BMJ Open

Diabetes

Research

\& Care

\title{
Population-based screen-detected type 2 diabetes mellitus is associated with less need for insulin therapy after 10 years
}

\author{
Rimke C Vos (1) , ${ }^{1,2}$ Henk den Ouden, ${ }^{2}$ Lois A Daamen, ${ }^{2}$ Henk J G Bilo, ${ }^{3}$ \\ Petra Denig, ${ }^{4}$ Guy E H M Rutten ${ }^{2}$
}

To cite: Vos $\mathrm{RC}$, den Ouden $\mathrm{H}$, Daamen LA, et al. Populationbased screen-detected type 2 diabetes mellitus is associated with less need for insulin therapy after 10 years. BMJ Open Diab Res Care 2020;8:e000949. doi:10.1136/ bmjdrc-2019-000949

Received 3 October 2019 Revised 10 January 2020 Accepted 7 February 2020

\section{Check for updates}

(c) Author(s) (or their employer(s)) 2020. Re-use permitted under CC BY-NC. No commercial re-use. See rights and permissions. Published by BMJ.

${ }^{1}$ Public Health and Primary Care/LUMC-Campus The Hague, LUMC, Leiden, The Netherlands

${ }^{2}$ Julius Center for Health Sciences and Primary Care, UMC Utrecht, Utrecht, The Netherlands

${ }^{3}$ Department of Internal Medicine, Isala Klinieken Locatie Weezenlanden, Zwolle, The Netherlands

${ }^{4}$ Department of Clinical Pharmacy and Pharmacology, UMCG, Groningen, The Netherlands

Correspondence to Dr Rimke C Vos; r.c.vos@lumc.nl

\section{ABSTRACT}

Introduction With increased duration of type 2 diabetes, most people have a growing need of glucose-lowering medication and eventually might require insulin. Presumptive evidence is reported that early detection (eg, by population-based screening) and treatment of hyperglycemia will postpone the indication for insulin treatment. A treatment legacy effect of population-based screening for type 2 diabetes of about 3 years is estimated. Therefore, we aim to compare insulin prescription and glycemic control in people with screen-detected type 2 diabetes after 10 years with data from people diagnosed with type 2 diabetes seven (treatment legacy effect) and 10 years before during care-as-usual.

Research design and methods Three cohorts were compared: one screen-detected cohort with 10 years diabetes duration (Anglo-Danish-Dutch study of Intensive Treatment in People with Screen-Detected Diabetes in Primary care (ADDITION-NL): $n=391$ ) and two care-as-usual cohorts, one with 7-year diabetes duration (Groningen Initiative to Analyze Type 2 Diabetes Treatment (GIANTT) and Zwolle Outpatient Diabetes project Integrating Available Care (ZODIAC): $n=4473$ ) and one with 10-year diabetes duration (GIANTT and ZODIAC: $\mathrm{n}=2660$ ). Insulin prescription (primary outcome) and hemoglobin A1c ( $\mathrm{HbA1C}$ ) of people with a known diabetes duration of 7 years or 10 years at the index year 2014 were compared using regression analyses. Results Insulin was prescribed in 10.5\% (10-year screen detection), $14.7 \%$ (7-year care-as-usual) and 19.0\% (10year care-as-usual). People in the 7-year and 10-year care-as-usual groups had a $1.5(95 \% \mathrm{Cl} 1.0$ to 2.1$)$ and 1.8 (95\% Cl 1.3 to 2.7$)$ higher adjusted odds for getting insulin prescribed than those after screen detection. Lower $\mathrm{HbA1C}$ values were found 10 years after screen detection (mean $50.1 \mathrm{mmol} / \mathrm{mol}(6.7 \%)$ vs $51.8 \mathrm{mmol} / \mathrm{mol}(6.9 \%)$ and $52.8 \mathrm{mmol} / \mathrm{mol}(7.0 \%))$, compared with 7 years and 10 years after care-as-usual ( $\mathrm{MD}_{\text {adiusted }}: 1.6 \mathrm{mmol} / \mathrm{mol}(95 \% \mathrm{Cl}$ 0.6 to 2.6$) ; 0.1 \%(95 \% \mathrm{Cl} 0.1$ to 0.2$)$ and $1.8 \mathrm{mmol} / \mathrm{mol}$ (95\% $\mathrm{Cl} 0.7$ to 2.9$)$; and $0.2 \%(95 \% \mathrm{Cl} 0.1$ to 0.3$)$ ). Conclusion Population-based screen-detected type 2 diabetes is associated with less need for insulin after 10 years compared with people diagnosed during care-asusual. Glycemic control was better after screen detection but on average good in all groups.

\section{INTRODUCTION}

In the Anglo-Danish-Dutch study of Intensive Treatment in People with Screen-Detected

\section{Significance of this study}

What is already known about this subject?

- With longer duration of type 2 diabetes, most people have a growing need of glucose-lowering medication and eventually require insulin.

- Early treatment of hyperglycemia postpones the indication for insulin treatment.

What are the new findings?

- Targeted population-based diabetes screening is associated with less need for insulin after 10 years compared with people diagnosed during care-as-usual.

- Glycemic control was slightly better after populationbased screen detection despite less insulin prescriptions.

How might these results change the focus of research or clinical practice?

- The earlier diabetes is diagnosed and an intervention is initiated, the more favorable results are possible.

Diabetes in Primary care (ADDITION) study, 300000 individuals with a high risk of type 2 diabetes were screened for type 2 diabetes, according to WHO 1999 criteria. ${ }^{12}$ Subsequently, in more than 3000 screen-detected patients, the effect of an intensive multifactorial treatment (IT) was compared with routine care (RC), according to national guidelines. ${ }^{3}$ After 5 years, a reduction in fatal and nonfatal cardiovascular disease (CVD) of $17 \%$ (n.s.) was found in favor of the IT group. A remarkable result was the finding that 5 years after screen detection, $37 \%$ and $45 \%$ of the participants with type 2 diabetes in the IT and RC groups did not use any glucose-lowering medication. ${ }^{3}$ After 5 years, the intervention stopped, and the subsequent treatment intensity was up to the general practitioner and the patient.

Results of two recent post hoc analysis with the ADDITION Denmark screening data indicated that after a follow-up of approximately 
10 years people diagnosed after population-based screening had a $21 \%$ significant lower risk on all-cause mortality and a $16 \%$ significant lower risk on CVD events, but no significant risk reduction on CVD mortality compared with unscreened controls. ${ }^{45}$ Overall, there was little benefit that could be directly related to the screening programme. In part, this was due to the high degree of opportunistic screening in the control group. Based on these results, it was suggested that if opportunistic screening has been well established in a healthcare system, population-based screening might not add additional health benefits. ${ }^{45}$ In the Netherlands, since two decades, general practitioners are recommended to screen people with a high risk on type 2 diabetes, during surgery hours. ${ }^{6}$ This opportunistic screening is likely established differentially across general practices. ${ }^{7}$

Targeted population-based screening as well as opportunistic screening detect people with type 2 diabetes relatively early in the course of their disease. At that time most of them will be asymptomatic. Although evidence is available that early detection is beneficial for disease control, the impact of population-based screening compared with care-as-usual (including not fully implemented opportunistic screening) needs to be explored.

With increased duration of type 2 diabetes, most people have a growing need of glucose-lowering medication and eventually require insulin. ${ }^{89}$ According to a computersimulated model based on the ADDITION data, it is speculated that the number of people on insulin therapy would be much higher if they were diagnosed during care-as-usual, an estimated (lead time of) 3-6years later. ${ }^{10}$ Moreover, presumptive evidence is reported that early treatment of hyperglycemia will postpone the indication for insulin treatment due to possible effects on $\beta$-cell function. ${ }^{11-13}$

Hence, we hypothesize that, irrespective of age at diagnosis, on the long run, the need for insulin therapy is less in people diagnosed with type 2 diabetes during targeted population-based screening (like in the ADDITION study) compared with people with diabetes diagnosed during care-as-usual. Therefore, we evaluated insulin prescription and glycemic control in Dutch participants from the ADDITION study (both IT and RC) 10 years after being diagnosed with type 2 diabetes. We compared these results with data from Dutch people with a known diabetes duration of 7 years (assuming a lead time of 3 years) and 10 years (since the exact lead time is unknown) from two 'care-as-usual' diabetes cohorts (Groningen Initiative to Analyze Type 2 Diabetes Treatment (GIANTT) and Zwolle Outpatient Diabetes project Integrating Available Care (ZODIAC)). ${ }^{14} 15$

\section{RESEARCH DESIGN AND METHODS}

\section{Subjects}

Participants with type 2 diabetes managed by general practitioners from three cohorts were included. One cohort was the ADDITION-NL cohort $(n=391)$, consisting of people diagnosed with type 2 diabetes between 2002 and 2003 after population-based screening. The two remaining cohorts included participants diagnosed with type 2 diabetes in usual care with opportunistic screening: the GIANTT and ZODIAC $^{14}$ cohorts $(n=3918) .{ }^{15}$ As stated above, opportunistic screening in the Netherlands was definitely not yet widely implemented at the start of the ADDITION study. ${ }^{15}$ The guidelines recommended to conduct 3 yearly fasting blood glucose testing in people aged $\geq 45$ years and one of the following characteristics: body mass index $(\mathrm{BMI}) \geq 27 \mathrm{~kg} / \mathrm{m}^{2}$, a family history of type 2 diabetes, being known with hypertension, dyslipidemia or CVD, a specific ethnicity (namely Turkish, Moroccan or Surinamese) and a history of gestational diabetes. In 2014, the ADDITION-NL cohort had a diabetes duration of $\sim 10$ years. It was estimated that population-based screening would detect people with type 2 diabetes approximately 3 years earlier. Therefore, participants from the care-as-usual cohorts were divided into two groups: people with a known type 2 diabetes duration of 7 years (assuming a lead time of 3 years) and 10 years (because the exact lead time is unknown), respectively.

\section{ADDITION-NL cohort}

The ADDITION-Europe study is a cluster-randomised, parallel-group trial evaluating the effect of early IT in people with screen-detected type 2 diabetes. The study was carried out among 343 general practices in Denmark, the UK and the Netherlands. Details of the study have been described previously. ${ }^{13}$ ADDITION-NL took place in the south western part of the Netherlands, identification of high risk for type 2 diabetes took place in all individuals aged 50-69 years and unknown with type 2 diabetes. High risk for diabetes type 2 was based on the modified version of the Symptom Risk Questionnaire used in the Hoorn study. The questionnaire contained questions on age, sex, BMI, family history of diabetes, frequent thirst, use of antihypertensive medication, shortness of breath, claudication and cycling. Individuals with a score of $\geq 4$ points were invited by their general practitioner for diabetes assessment, using a random blood glucose test, a fasting blood glucose test and an oral glucose tolerance test. The WHO 1999 criteria for type 2 diabetes were used for diagnosis. ${ }^{2}$

Exclusion criteria were illness with a life expectancy less than 1year and psychiatric disorders invalidating informed consent or impeding study participation. Practice-level randomization was carried out in 79 general practices for providing either care-as-usual, according to the 1999 guidelines of the Dutch College of General Practitioners ${ }^{6}$ or IT according to the ADDITION-NL protocol. Randomization was performed according to stratification of practice organization (number of patients: 215 IT and $176 \mathrm{RC}) .{ }^{16}$ In the 5 years following randomization, all patients with screen-detected type 2 diabetes were treated according to the randomly allocated treatment protocol. General practitioners and nurses in practices randomized to intensive multifactorial treatment were 
motivated during educational meetings up to twice a year, for providing strict target-driven pharmacological treatment of hyperglycemia as well as promoting healthy lifestyles. After 5 years, the trial ended, and after the trial period, all patients were most likely treated according to the revised Dutch guidelines. ${ }^{17}$

\section{GIANTT cohort}

The GIANTT study started in 2004 in the northern part of the Netherlands as a prospective observational study for people with type 2 diabetes with the aim to improve diabetes care through yearly feedback information and advice for participating healthcare professionals. The database contains anonymized longitudinal information retrieved from electronic medical records of general practitioners and is maintained by the University Medical Center Groningen. ${ }^{18}$ These records include medical history, prescription data, routine laboratory test results and physical examinations of people with type 2 diabetes (no age restrictions), who did not opt-out for the use of their medical data for scientific research. For scientific research with anonymous data, this opt-out regulation is offered in the Netherlands as option instead of providing full informed consent. Medical history contains date of diabetes diagnosis and comorbidity data based on the International Classification of Primary Care or text descriptions that are coded manually. Data of people with a general practitioner-confirmed diagnosis of type 2 diabetes, exclusively treated in primary care, and a duration of 7 years or 10 years at the index year 2014 were included in the current study.

\section{ZODIAC cohort}

The ZODIAC study started in 1998 in the eastern part of the Netherlands as a prospective observational study for people with type 2 diabetes. ${ }^{19}$ Study goal was improvement of standard care through addition of extra caregivers, education and at least yearly feedback information and advice for the participating healthcare professionals. At inclusion, the individuals participating in the ZODIAC study were known with or newly diagnosed with type 2 diabetes and exclusively treated in primary care; at inclusion, they provided informed consent for the use of their anonymized data for research purposes. Individuals who were already treated for their diabetes in secondary care, individuals with a very short life expectancy and individuals with insufficient cognitive abilities were excluded. The number of participating general practitioners increased from 53 in 1998 to 459 in 2008. Patient numbers increased from 1622 to 27438 in this time frame, and nowadays even more than 40000 patients are participating.

\section{Design of the present comparison}

Cohort study, with 2014 as index year. Within the three cohorts, people were treated according to the same national guidelines, with the exception of the ADDITION-IT group. In this group, patients have been treated more strictly during the first 5 years after diagnosis. The frequency of diabetes monitoring, namely four times per year, was equal in all cohorts, all groups and all periods.

\section{Therapy targets}

At start of the ADDITION study, hemoglobin A1c (HbA1c) levels were targeted at $<64 \mathrm{mmol} / \mathrm{mol}(8.5 \%)$ and $<53 \mathrm{mmol} / \mathrm{mol}(7.0 \%)$ for the $\mathrm{RC}$ group and IT group, respectively. Moreover, in the IT arm, glucoselowering medication should be started as soon as the HbAlc level passed $47 \mathrm{mmol} / \mathrm{mol}$ (6.5\%). Since 2006, the revised Dutch national guidelines set an HbAlc of $<53 \mathrm{mmol} / \mathrm{mol}(7 \%)$ as target. ${ }^{17}$ In 2013 , less strict targets were set for people above 70 years and with a diabetes duration longer than 10 years. ${ }^{20}$

\section{Data assessment}

Subject characteristics during the index year: age, sex and disease control characteristics (HbA1c, lipoproteins (serum) - low-density lipoprotein (LDL) cholesterol, systolic blood pressure (SBP), BMI and smoking status) were all retrieved from the electronic medical records of the general practitioners. Assessment dates: GIANTT: January-July, ADDITION: January-December, ZODIAC: January-December, with value and date of last measurement available in the index period.

\section{Glucose-lowering medication}

Data of glucose-lowering medication prescribed in the index year were collected from the electronic medical records of the general practitioners using The Anatomical Therapeutic Chemical Classification System code: metformin (A10BA02), sulfonylurea (SU) (A10BB, A10 $\mathrm{BB} 12$ and A10BB09), insulin (A10A), and other oral glucose-lowering agents (A10BG, A10BK and A10BJ). Glucose-lowering mediation was categorized according to the Dutch treatment algorithm, with the following steps: (1) lifestyle only; (2) metformin; (3) metformin+SU and (4) insulin \pm oral agents, using other oral blood glucoselowering agents or insulin monotherapy in case of intolerance or contraindications for metformin, SU or insulin. ${ }^{20}$

\section{Outcomes}

\section{Primary outcome}

The primary outcome was prescription of any insulin (either monotherapy or in addition to oral agents) during the index year.

\section{Secondary outcome}

The secondary outcome was glycemic control (HbAlc level) during the index year

\section{Statistical analyses}

Data of study participants from the former RC and IT groups of the ADDITION-NL cohort were merged and analyzed as one group. Descriptive statistics were performed using SPSS V.24. Categorical variables are reported as counts and percentages, continuous variables as means with $\mathrm{SD}$ or medians with IQR for non-normally 
distributed variables. Differences with regard to subject characteristics between the population-based screening and care-as-usual groups were determined by analysis of variance for continuous variables and by $\chi^{2}$ tests for categorical variables. Logistic regression analysis was used to evaluate the difference in insulin prescription 10 years after screen detection compared with care-as-usual, adjusted for the following confounders: age, sex, HbA1c, SBP, LDL cholesterol, BMI and smoking, with cohort as fixed factor. To determine the differences in HbAlc linear regression analyses was used, adjusted for age, sex, $\mathrm{BMI}$ and glucose-lowering medication according to the abovementioned classification. All confounders had $<7 \%$ missing data, except for BMI (10.2\% missings) and LDL (13.8\% missings). To evaluate the impact of missing data, we performed multiple imputation, with a 10 imputed dataset under the assumption 'missing at random' as sensitivity analysis. To evaluate the impact of populationbased screening without the effect of multifactorial intensive treatment during the first 5 years, the ADDITION-NL RC group only was compared with the 7 years and 10 years care-as-usual groups as a sensitivity analysis.

\section{RESULTS}

Data of 7524 people with type 2 diabetes and data on glucose-lowering medication were included; 391 participants had population-based screen-detected diabetes (ADDITION-NL cohort) and 7133 people were diagnosed with type 2 diabetes during care-as-usual (GIANTT and ZODIAC combined): 4473 with 7 years $(\mathrm{n}=2014$ GIANTT, $\mathrm{n}=2459$ ZODIAC) and 2660 with 10-year diabetes duration ( $\mathrm{n}=1201$ GIANTT, $\mathrm{n}=1459$ ZODIAC). Subject characteristics of the three groups are presented in table 1. People with screen-detected diabetes were on average older and more often male compared with people from the two other cohorts. Ten years after their screen detection, people showed a lower SBP $(135 \mathrm{~mm} \mathrm{Hg}$ vs $138 \mathrm{~mm}$ $\mathrm{Hg}$ in both care-as-usual groups) and LDL-cholesterol levels $(2.1 \mathrm{mmol} / \mathrm{L}$ vs $2.5 \mathrm{mmol} / \mathrm{L}$ in both care-as-usual groups) but a higher BMI: $30.4 \mathrm{~kg} / \mathrm{m}^{2}$ vs $29.8 \mathrm{~kg} / \mathrm{m}^{2}$ in the 7 years and $29.4 \mathrm{~kg} / \mathrm{m}^{2}$ in the 10 years group.

\section{Insulin prescription}

Insulin prescriptions in people with screen-detected diabetes and in people known with diabetes for 7 years and 10 years during care-as-usual were $10.5 \%, 14.8 \%$ and $19.0 \%$, respectively (table 1). After adjustment for confounders, people diagnosed 7 years and 10 years before the index year during care-as-usual had a 1.5 (95\% CI 1.0 to 2.1 ) and 1.8 (95\% CI 1.3 to 2.7) times higher chance of getting insulin prescribed compared with those with screen-detected diabetes. Similar results were found after multiple imputation (table 2). Comparing only the ADDITION-NL RC group with the care-as-usual groups, the observed point estimates (mean difference) remained similar in both groups. The broader $95 \%$ CIs

\begin{tabular}{|c|c|c|c|c|c|}
\hline & $\begin{array}{l}\text { ADDITION-NL } \\
(\mathrm{n}=391)\end{array}$ & $\begin{array}{l}\text { 7-years diabetes } \\
\text { diagnosis }(n=4473)\end{array}$ & $\begin{array}{l}\text { 10-year diabetes } \\
\text { diagnosis }(n=2660)\end{array}$ & F-test $/ \chi^{2}$ & $P$ value \\
\hline Age (years) & $71.6(5.3)$ & $68.4(11)$ & $69.7(10.6)$ & 22.9 & $<0.01$ \\
\hline Sex, male $(n, \%)$ & $208(52.7)$ & $2266(50.5)$ & $1272(47.8)$ & 6.2 & 0.04 \\
\hline Smoking, yes (n, \%) & $52(14.2)$ & $706(16.4)$ & 407 (15.9) & 1.2 & 0.55 \\
\hline $\operatorname{BMI}\left(\mathrm{kg} / \mathrm{m}^{2}\right)$ & $30.4(6.7)$ & $29.8(5.3)$ & $29.4(5.2)$ & 7.1 & $<0.01$ \\
\hline $\mathrm{HbA1c}(\%)$ & $6.7(0.9)$ & $6.9(0.9)$ & $7.0(1.0)$ & $N A^{*}$ & $N A^{*}$ \\
\hline $\mathrm{HbA} 1 \mathrm{c}(\mathrm{mmol} / \mathrm{mol})$ & $50.1(9.8)$ & $51.8(10.1)$ & $52.8(10.4)$ & $N A^{*}$ & $N A^{*}$ \\
\hline $\mathrm{SBP}(\mathrm{mm} \mathrm{Hg})$ & $135(15.4)$ & $138(16.1)$ & $138(16.6)$ & 4.2 & 0.02 \\
\hline $\mathrm{DBP}(\mathrm{mm} \mathrm{Hg})$ & $74(9.4)$ & $77(9.2)$ & $76.1(9.1)$ & 13.9 & $<0.01$ \\
\hline LDL (mmol/L) & $2.1(0.9)$ & $2.5(0.9)$ & $2.5(0.9)$ & 33.7 & $<0.01$ \\
\hline Glucose-lowering medication & & & & $N A^{*}$ & $N A^{*}$ \\
\hline Lifestyle only & $72(18.4)$ & $795(17.7)$ & $380(14.3)$ & & \\
\hline Metformin & $116(29.7)$ & $1501(33.4)$ & $736(27.7)$ & & \\
\hline Metformin+SU & $138(35.3)$ & $1257(28.0)$ & 848 (31.9) & & \\
\hline Other oral antidiabetic agents & $24(6.1)$ & $275(6.1)$ & $192(7.2)$ & & \\
\hline Metformin and/or SU+insulin & $26(6.6)$ & $492(11.0)$ & $377(14.2)$ & & \\
\hline Insulin monotherapy & $12(3.1)$ & $138(3.1)$ & $103(3.9)$ & & \\
\hline $\begin{array}{l}\text { Insulin+other oral diabetic } \\
\text { agents }\end{array}$ & $3(0.8)$ & $31(0.7)$ & $24(0.9)$ & & \\
\hline
\end{tabular}

*Outcome measures (see table 2).

ADDITION, Anglo-Danish-Dutch study of Intensive Treatment in People with Screen-Detected Diabetes in Primary care; BMI, body mass index; DBP, Diastolic Blood Pressure; HbA1c, hemoglobin A1c; LDL, low-density lipoprotein; SBP, systolic blood pressure; SU, sulfonylurea. 
Table 2 Chance in getting insulin prescribed and mean difference in $\mathrm{HbA1c}$ for people diagnosed during care-as-usual compared with those with screen-detected T2DM

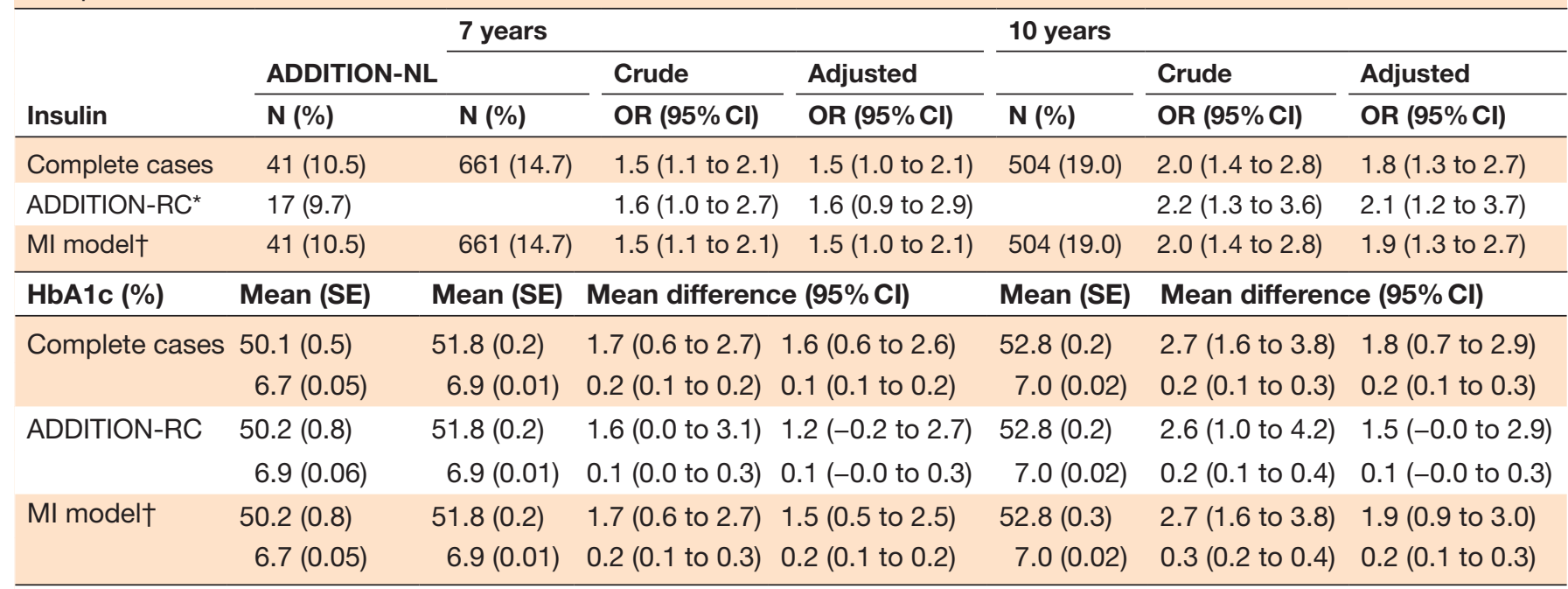

Model on insulin prescription adjusted for age, sex, HbA1c, SBP, LDL-cholesterol, BMI and smoking; model with HbA1c as outcome,

adjusted for sex, age, BMl and glucose-lowering medication.

*Sensitivity analysis with only care-as-usual group (RC) from ADDITION.

†Sensitivity analysis with multiple imputation.

ADDITION, Anglo-Danish-Dutch study of Intensive Treatment in People with Screen-Detected Diabetes in Primary care ; BMI, body mass

index; HbA1c, hemoglobin A1c; LDL, low-density lipoprotein; SBP, systolic blood pressure; T2DM, type 2 diabetes mellitus.

resulted in a non-significant OR compared with the 7 years group $\left(\mathrm{OR}_{\text {adiusted }}=1.6\right.$ (95\% CI 0.9 to 2.9$)$. The OR compared with the 10 years group $\left(\mathrm{OR}_{\text {adjusted }}=2.1(95 \% \mathrm{CI}\right.$ 1.2 to 3.7 ) was larger and remained significant (table 2 ).

\section{Glycemic control}

Ten years after their screen detection of type 2 diabetes diagnosis, people showed on average a slightly lower HbAlc level compared with those with a 7-year and 10-year diabetes diagnosis based on care-as-usual, namely $50.1 \mathrm{mmol} / \mathrm{mol}(6.7 \%)$ versus $51.8 \mathrm{mmol} / \mathrm{mol}(6.9 \%)$ in those with 7 -year and $52.8 \mathrm{mmol} / \mathrm{mol}(7.0 \%)$ with 10 -year diabetes diagnosis. The adjusted mean differences between people with screen-detected diabetes and those diagnosed 7 years and 10 years before during careas-usual were $1.6 \mathrm{mmol} / \mathrm{mol}(95 \% \mathrm{CI} 0.6$ to 2.6$)$ ( $0.1 \%$ (95\% CI 0.1 to 0.2$)$ ) and $1.8 \mathrm{mmol} / \mathrm{mol}$ (95\% CI 0.7 to 2.9) $(0.2 \%$ (95\% CI 0.1 to 0.3$))$, respectively. Similar results were found after multiple imputation (table 2). Comparing only the ADDITION-NL RC group with the care-as-usual groups, the observed mean differences remained similar and were borderline significant with adjusted mean differences of $1.2 \mathrm{mmol} / \mathrm{mol}(95 \% \mathrm{CI}-0.2$ to 2.7$)(0.1 \%(95 \% \mathrm{CI}-0.0$ to 0.3$))$ and $1.5 \mathrm{mmol} / \mathrm{mol}$ (95\% CI -0.0 to 2.9$)(0.1 \%$ (95\% CI -0.0 to 0.3$)$ (table 2$)$.

\section{CONCLUSION}

In this study, we compared 10-year follow-up data after population-based diabetes screening with both 7-year and 10-year follow-up data from care-as-usual. We assumed a lead time of 3 years, based on the results of a computersimulated model based on the ADDITION Europe data. ${ }^{8}$ Since the exact lead time of population-based screening is unknown, we also compared a similar prescribing period (10 years). Our results show that population-based diabetes screening is associated with less need for insulin therapy compared with care-as-usual, which may include but does not guarantee opportunistic screening, in combination with a slightly better long-term glycemic control. Although our findings might seem obvious, because individuals diagnosed during opportunistic screening are likely to have a longer undiagnosed period, they are nevertheless clinically relevant. The ADDITION-DK study suggested that population-based screening has no additional benefits above opportunistic screening on risk reduction on CVD mortality. ${ }^{45}$ However, our results indicate that population-based screening might result in a delayed need for insulin, which may influence the discussion on population-based versus opportunistic screening for type 2 diabetes.

In people with screen-detected type 2 diabetes, the HbAlc may be lower compared with opportunistic screening and particularly compared with people with symptoms of high glucose levels. In a former study, a high HbA1c at time of diagnosis was strongly associated with early initiation of insulin and poorer glycemic control in the future, independent of the selected glucose-lowering therapy. ${ }^{21}$ Therefore, it might be speculated that the delayed need for insulin in the screen-detected people with type 2 diabetes is at least partially related to a difference in HbA1c level at time of diagnosis. Unfortunately, in the GIANNT and ZODIAC cohorts, data on HbAlc at time of diagnosis were not available in the majority of patients. The UK Prospective Diabetes Study demonstrated that a higher glucose level at diagnosis was associated with a decreased likelihood of achieving HbAlc 
targets and, subsequently, progressive requirement for pharmacological treatment. ${ }^{922}$ However, earlier intensive treatment is more likely to result in the achievement of treatment targets, which are more likely thereafter to be maintained. This explanation is in line with the results of a retrospective analysis of the UK Clinical Practice Research Datalink database Study. This study showed that the likelihood of attaining glycemic control was $22 \%$ and $28 \%$ lower for patients in the intermediate $(12-<24$ months) and late (24-<36 months) intensification groups, respectively, compared with those whose therapy had been intensified within 12 months. ${ }^{23}$

Considering the type of glucose-lowering medication prescriptions, according to both the ADDITION protocol and the Dutch diabetes guidelines since 2006, metformin as well as SU should be prescribed to the maximally tolerable dose before insulin is prescribed, taken into account a contraindication of metformin for people with an estimated glomerular filtration rate (eGFR) $<30 \mathrm{~mL} /$ $\min / 1.73 \mathrm{~m}^{2} .{ }^{20}$ Our results could also be partly due to a difference in prescribing behavior of the physicians in the different cohorts, both in terms of type of glucoselowering medication and in dosages. Alternatively, the people's age at the time of diagnosis could also play a role; people from the ADDITION-NL cohort were, as a result of the inclusion criteria, on average 3 years older when they were diagnosed with type 2 diabetes. It could be that early detection and a diagnosis of type 2 diabetes at older age are associated with a delayed need for more intensive treatment. Finally, the method used for diagnosing type 2 diabetes differs between the cohorts. In the ADDITION-NL cohort, it was based on stepwise screening including risk assessment using the Symptom Risk Questionnaire, followed by random and/or fasting glucose testing and additional an OGTT if needed. In the ADDITION-NL cohort, HbA1c has not been used as a diagnostic marker. In care-as-usual, about $10 \%$ of the patients are symptomatic at diagnosis after which a random glucose value will suffice according to the guidelines from the Dutch College of General Practitioners. In the vast majority of asymptomatic people who are diagnosed with type 2 diabetes by care-as-usual, at least one fasting glucose measure in a certified laboratory will have been taken. According to the above-mentioned guidelines, general practitioners were not advised to use HbA1c as a diagnostic marker. Altogether, however, the different diagnosing strategies all result in a diagnosis of diabetes that is considered reliable. So, whereas the diagnostic process and the HbA1c level at diagnosis will likely to be different between the cohorts, they are intrinsically linked to the difference between population-based screening and care-as-usual. Therefore, adjusting for these factors would not be justified, and the results of this study allow conclusions based on the time of detection.

Strengths of this study are the availability of detailed data on glycemic control and glucose-lowering medication over a follow-up period of 10 years after populationbased screen detection of type 2 diabetes and data from care-as-usual from the same healthcare system. Several limitations should be mentioned. First, the design of our study does not allow causal interpretation; we adjusted for some patient differences, but bias and confounding are possible. Second, we included three Dutch cohorts from different regions with may be different patients' socioeconomic status, ethnicity and willingness to participate. Finally, we assumed a lead time of 3 years with population-based screen detection, but the higher age at diagnosis in the ADDITION-NL cohort suggests this may not be true.

In conclusion, 10 years after screen-detected type 2 diabetes according to $\mathrm{WHO}$ criteria, a lower number of people are in need of insulin compared with individuals diagnosed during care-as-usual, in combination with a slightly better glycemic control. This suggests that the earlier diabetes is diagnosed and an intervention is initiated, the more favorable results are possible.

Contributors GEHMR and RCV designed the study. RCV analyzed the data and drafted the manuscript. RCV and HdO coordinated the data collection of the Anglo-Danish-Dutch study of Intensive Treatment in People with Screen-Detected Diabetes in Primary care-NL data. LAD and $\mathrm{HdO}$ were involved in drafting and analysing previous versions of the manuscript. HJGB is responsible for the Zwolle Outpatient Diabetes project Integrating Available Care data and PD for the Groningen Initiative to Analyze Type 2 Diabetes Treatment data. All authors read and approved the final manuscript. RCV is the guarantor of this work and, as such, had full access to all the data in the study and takes responsibility for the integrity of the data and the accuracy of the data analysis.

Funding The authors have not declared a specific grant for this research from any funding agency in the public, commercial or not-for-profit sectors.

Competing interests None declared.

Patient consent for publication Not required.

Ethics approval The Medical Ethical Committee of the University Medical Center Utrecht, the Netherlands, approved the study and all participants gave their informed consent.

Provenance and peer review Not commissioned; externally peer reviewed.

Data availability statement Data are available on reasonable request.

Open access This is an open access article distributed in accordance with the Creative Commons Attribution Non Commercial (CC BY-NC 4.0) license, which permits others to distribute, remix, adapt, build upon this work non-commercially, and license their derivative works on different terms, provided the original work is properly cited, appropriate credit is given, any changes made indicated, and the use is non-commercial. See: http://creativecommons.org/licenses/by-nc/4.0/.

ORCID ID

Rimke C Vos http://orcid.org/0000-0003-1074-6255

\section{REFERENCES}

1 van den Donk M, Sandbaek A, Borch-Johnsen $\mathrm{K}$, et al. Screening for type 2 diabetes. lessons from the ADDITION-Europe study. Diabet Med 2011;28:1416-24.

2 World Health Organization. Cardiovascular diseases (CVDs) fact sheet $N^{\circ} 317,2015$. Available: http://www.who.int/diabetes/ publications/diagnosis_diabetes2006/en/index.html

3 Griffin SJ, Borch-Johnsen K, Davies MJ, et al. Effect of early intensive multifactorial therapy on 5-year cardiovascular outcomes in individuals with type 2 diabetes detected by screening (ADDITIONEurope): a cluster-randomised trial. Lancet 2011;378:156-67.

4 Simmons RK, Griffin SJ, Witte DR, et al. Effect of population screening for type 2 diabetes and cardiovascular risk factors on mortality rate and cardiovascular events: a controlled trial among 1,912,392 Danish adults. Diabetologia 2017;60:2183-91.

5 Simmons RK, Griffin SJ, Lauritzen T, et al. Effect of screening for type 2 diabetes on risk of cardiovascular disease and mortality: a 
controlled trial among 139,075 individuals diagnosed with diabetes in Denmark between 2001 and 2009. Diabetologia 2017;60:2192-9.

6 Rutten G, Verhoeven S, Heine RJ, et al. NHG-standaard diabetes mellitus type 2. Huisarts Wet 1999;42:67-84.

7 Janssen PGH, Gorter KJ, Stolk RP, et al. Do characteristics of practices and general practitioners influence the yield of diabetes screening in primary care? the addition Netherlands study. Scand J Prim Health Care 2008;26:160-5.

8 García-Pérez L-E, Alvarez M, Dilla T, et al. Adherence to therapies in patients with type 2 diabetes. Diabetes Ther 2013;4:175-94.

9 Turner RC, Cull CA, Frighi V, et al. Glycemic control with diet, sulfonylurea, metformin, or insulin in patients with type 2 diabetes mellitus: progressive requirement for multiple therapies (UKPDS 49). UK prospective diabetes study (UKPDS) group. JAMA 1999;281:2005-12.

10 Herman WH, Ye W, Griffin SJ, et al. Early detection and treatment of type 2 diabetes reduce cardiovascular morbidity and mortality: a simulation of the results of the Anglo-Danish-Dutch study of intensive treatment in people with screen-detected diabetes in primary care (ADDITION-Europe). Diabetes Care 2015;38:1449-55

11 Holman RR, Paul SK, Bethel MA, et al. 10-Year follow-up of intensive glucose control in type 2 diabetes. N Engl J Med 2008;359:1577-89.

12 ACCORD Study Group. Nine-Year effects of 3.7 years of intensive glycemic control on cardiovascular outcomes. Diabetes Care 2016;39:701-8.

13 Punthakee Z, Miller ME, Simmons DL, et al. Durable change in glycaemic control following intensive management of type 2 diabetes in the Accord clinical trial. Diabetologia 2014;57:2030-7.

14 de Vries ST, Voorham J, Haaijer-Ruskamp FM, et al. Potential overtreatment and undertreatment of diabetes in different patient age groups in primary care after the introduction of performance measures. Diabetes Care 2014;37:1312-20.
15 van Hateren KJJ, Drion I, Kleefstra N, et al. A prospective observational study of quality of diabetes care in a shared care setting: trends and age differences (ZODIAC-19). BMJ Open 2012;2:1.

16 Janssen PG, Gorter KJ, Stolk RP, et al. Randomised controlled trial of intensive multifactorial treatment for cardiovascular risk in patients with screen-detected type 2 diabetes: 1-year data from the addition Netherlands study. Br J Gen Pract 2009;59:43-8.

17 Rutten G, WJCd G, Nijpels G, et al. NHG-standaard diabetes mellitus type 2 (second revision). Huisarts Wet 2006;49:137-52.

18 Voorham J, Haaijer-Ruskamp FM, van der Meer K, et al. Identifying targets to improve treatment in type 2 diabetes; the Groningen initiative to aNalyse type 2 diabetes treatment (GIANTT) observational study. Pharmacoepidemiol Drug Saf 2010;19:1078-86.

19 Ubink-Veltmaat LJ, Bilo HJG, Groenier KH, et al. Shared care with task delegation to nurses for type 2 diabetes: prospective observational study. Neth J Med 2005;63:103-10.

20 Rutten G, WJCd G, Nijpels G, et al. NHG-standaard diabetes mellitus type 2. NHG-standaarden (third reversion). Huisarts Wet 2013;2:1-49.

21 Spoelstra JA, Stolk RP, Klungel $\mathrm{OH}$, et al. Initiation of glucoselowering therapy in type 2 diabetes mellitus patients in general practice. Diabet Med 2004;21:896-900.

22 Colagiuri S, Cull CA, Holman RR, et al. Are lower fasting plasma glucose levels at diagnosis of type 2 diabetes associated with improved outcomes?: U.K. prospective diabetes study 61. Diabetes Care 2002;25:1410-7.

23 Desai U, Kirson NY, Kim J, et al. Time to treatment intensification after monotherapy failure and its association with subsequent glycemic control among 93,515 patients with type 2 diabetes. Diabetes Care 2018;41:2096-104. 